\title{
Novel triple lumen catheter for ERCP tissue diagnosis
}

\section{(ㄷ)(이우}

\author{
Authors \\ Martin Goetz, Nisar P. Malek \\ Institution \\ Innere Medizin I, Universitätsklinikum Tübingen, Tübingen, \\ Germany \\ submitted 19.9.2017 \\ accepted after revision 19.1.2018

\section{Bibliography} \\ DOI https://doi.org/10.1055/a-0591-2740 | \\ Endoscopy International Open 2018; 06: E984-E988 \\ (C) Georg Thieme Verlag KG Stuttgart · New York \\ ISSN 2364-3722 \\ Corresponding author \\ Martin Goetz, MD, Innere Medizin I, Universitätsklinikum \\ Tübingen, 72076 Tübingen, Germany \\ Fax: +49-7071-2925034 \\ martin.goetz@med.uni-tuebingen.de
}

\section{ABSTRACT}

Introduction The ideal endoscopic retrograde cholangiopancreatography (ERCP) tissue sampling technique should be simple, inexpensive, and yield sufficient tissue for histology while permitting contrast-guided targeting under fluorescence and multiple passes into the bile duct. Current techniques do not fulfill these requirements comprehensively. We designed a catheter for optimized targeting of large intrabiliary biopsies.

Methods A 3.5-mm sterile, single-use prototype catheter was developed, with three independent lumina extending continuously to the distal tip for three distinct functions to be available simultaneously: passage of large volume biopsy forceps $(1.8 \mathrm{~mm})$, wire guidance $\left(0.035^{\prime \prime}\right)$, and contrast injection.

Results First clinical use in five patients (four transpapillary, one percutaneous access) allowed good intrabiliary manipulation in $4 / 5$ patients. Contrast-guided sampling provided adequate specimens. No adverse events were noted.

Discussion Technical aspects and preliminary clinical data for the novel triple lumen biopsy catheter are encouraging. The catheter allowed wire guidance and contrast application to guide sampling of larger pieces of tissue. In perspective, the working channel could host any accessory for targeted intrabiliary diagnosis and therapy, and catheter use is not limited to bile ducts.

\section{Introduction}

The ideal endoscopic retrograde cholangiopancreatography (ERCP) tissue sampling technique should be safe, simple, inexpensive, and yield sufficient tissue for histology and immunohistochemistry or at least adequate cellularity for cytology. The currently available techniques do not fulfill these requirements comprehensively. Brush cytology is the standard in most departments due to its high specificity, relative ease-ofuse, safety, and low cost. However, its sensitivity for cancer is limited by only superficial tissue exfoliation and yield of cytologic rather than histological specimens. A sensitivity of $42 \%$ was reported in a recent review [1]. Freehand endobiliary forceps biopsy sampling is technically more challenging. A standard or pediatric biopsy forceps is advanced across the papilla, usually after sphincterotomy, toward the suspected area of interest. Biopsy sampling provides larger and fewer superficial pieces of tissue for histological assessment. Sensitivity seems to be higher $(60 \%)$ [1] than for brush cytology. The optimal number of biopsies has not been established, but a minimal number of three specimens has been suggested [2,3]. Adverse events are uncommon, but may include bleeding and perforation, especially in multiple passes with a stiff forceps [4]. Cholangioscopic biopsy intuitively seems the optimal sampling method, with accuracy rates for biopsies from single operator cholangioscopy ranging from $60.6 \%$ [5] to $89 \%$ [6]. It requires more expertise and is a costly procedure but it adds direct visualization and visual targeting of biopsies. Biopsies are small (forceps, $1.2 \mathrm{~mm}$ diameter) and multiple passes are required [7]. Our aim was the design and first clinical assessment of a simple-to-use catheter for optimized targeting of large intrabiliary biopsies.

\section{Methods}

\section{Catheter design}

A catheter was designed to harbor three independent lumina extending continuously to ports at the distal tip in order to provide three distinct functions to be available simultaneously: first, wire guidance to facilitate navigation across the papilla and along the bile duct and hilar region; second, contrast injec- 

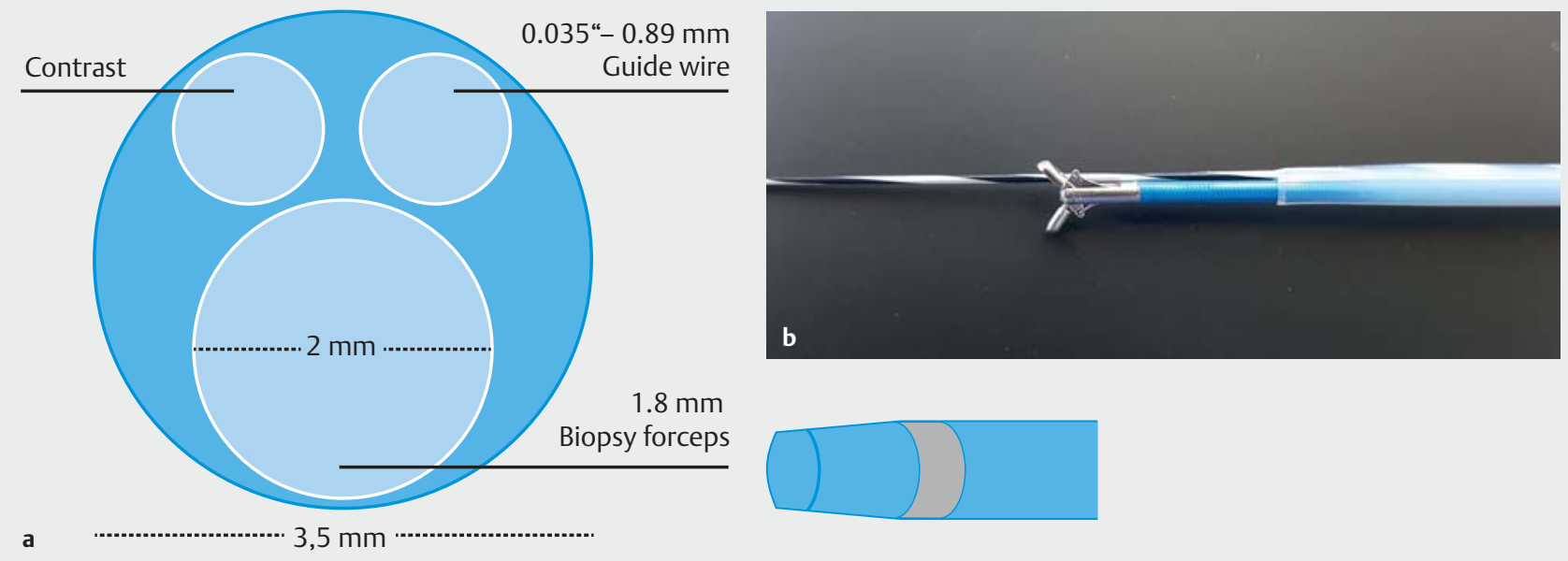

b
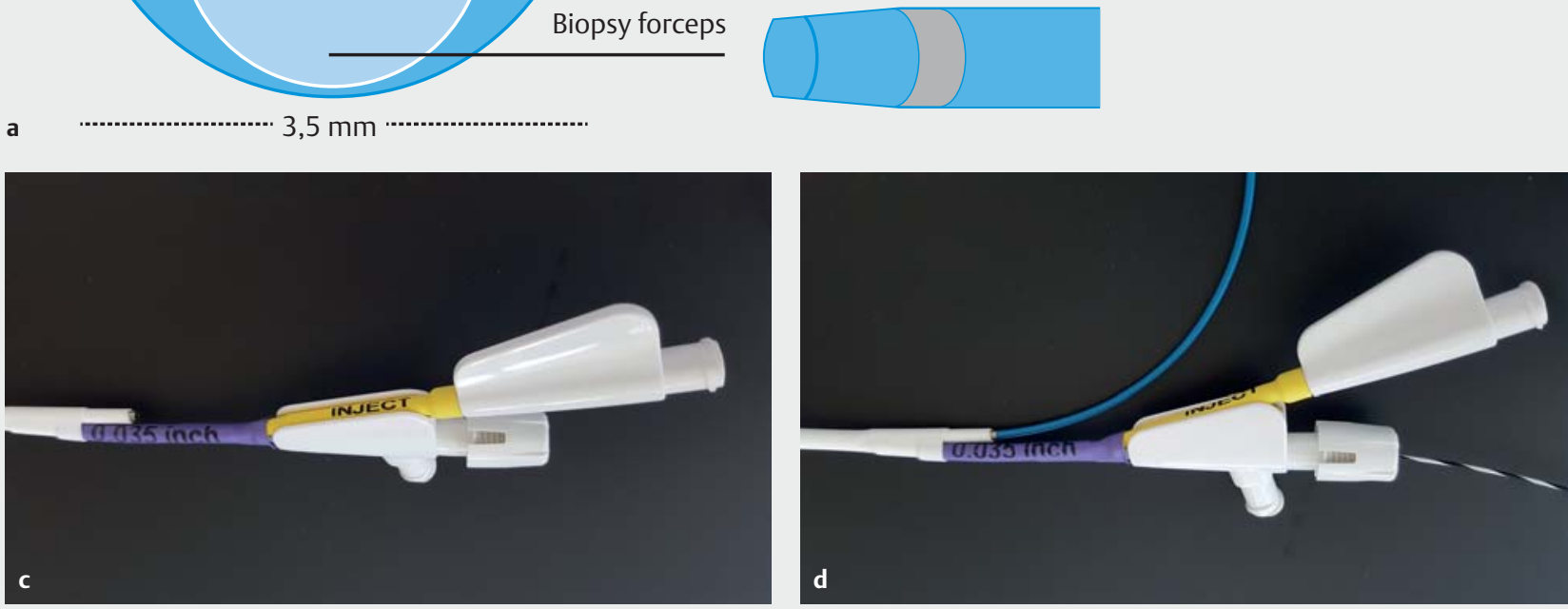

- Fig. 1 Catheter tip and hand piece with guidewire and biopsy forceps. a Schematic cross-section of the catheter with three lumina and tapered catheter tip with radio-opaque ring marker. b Catheter tip with $1.8 \mathrm{~mm}$ biopsy forceps and $0.035^{\prime \prime}$ guidewire. c Catheter handpiece without and $\mathbf{d}$ with biopsy forceps and guidewire, injection port in between the former two.

tion for exact verification of the region of interest under fluoroscopy while obtaining a biopsy; and third, passage of large volume biopsy forceps.

Therefore, a 3.5-mm sterile, single-use plastic catheter prototype was produced hosting a 2-mm working channel and two additional channels for a $0.035^{\prime \prime}$ guidewire and contrast injection, respectively ( $\mathbf{F i g . 1}$ ). A metallic ring was placed $2 \mathrm{~cm}$ from the tip. A 1.8-mm biopsy forceps (Medwork BIO1-C4-18260 , working length $2.6 \mathrm{~m}$ ) was used for tissue sampling. Catheters were produced by Medwork (Hoechstadt/Aisch, Germany) in a GMP-certified facility (Certification according to ISO 13485). Informed consent was obtained from each patient. A patent was filed by the author (MG), the Department of Gastroenterology (Innere Medizin I), and the University of Tübingen (PCT/EP2016/070530).

\section{Bile duct biopsies}

Catheter prototypes were used for transpapillary biopsies during endoscopic retrograde cholangiography (ERC, $>$ Fig. 2) in four patients and for percutaneous biopsy in one patient ( $\triangleright$ Fig.3). All patients underwent ERCP or percutaneous transhepatic cholangiographic drainage (PTCD) with indication for tissue sampling by other methods and provided informed consent for additional catheter use.

\section{Results}

\section{Performance of the catheter}

For the first three prototypes, catheters with a straight, nontapered tip were built. In one patient with a papillary adenoma extending into the distal common bile duct, the catheter could not traverse the papilla. Therefore, the subsequent two prototypes were modified to provide a tapered, conical tip to facilitate transpapillary access. In one patient, owing to a difficult duodenoscope position, the biopsy forceps advancement through the catheter across the angulation at the Albarran lever had to be combined with simultaneous brief catheter advancement, before the forceps was advanced independently and relative to the catheter to allow biliary access. Indications for tissue sampling included five stenoses in three patients with inflammatory bile duct disease (primary sclerosing cholangitis (PSC), recurrent pyogenic cholangitis [RPC]); undetermined stenosis in one patient; and malignant in-stent stenosis in another (percutaneous access). Stenoses were located in the distal-mid common bile duct (CBD) (five stenoses) or perihilar region (two stenoses). In one patient, a mid-CBD stenosis was well accessible, but a second stenosis in the cranial portion of the left hepatic duct was not reached due to difficulties with 

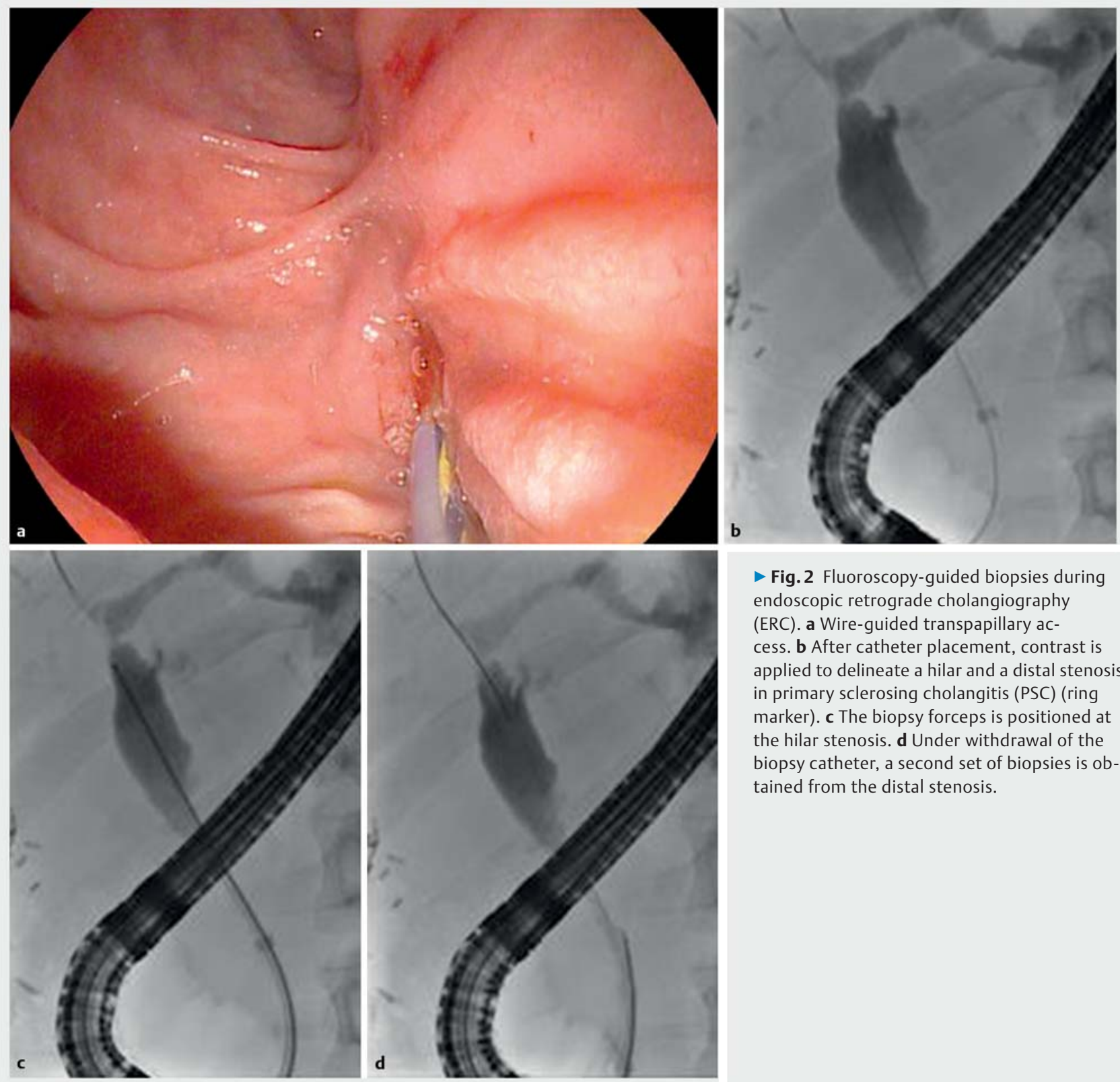

- Fig. 2 Fluoroscopy-guided biopsies during endoscopic retrograde cholangiography (ERC). a Wire-guided transpapillary access. b After catheter placement, contrast is applied to delineate a hilar and a distal stenosis in primary sclerosing cholangitis (PSC) (ring marker). c The biopsy forceps is positioned at the hilar stenosis. $\mathbf{d}$ Under withdrawal of the biopsy catheter, a second set of biopsies is obtained from the distal stenosis.

the catheter not able to follow the guidewire across a sharply curved bile duct course.

\section{Biopsy adequacy}

Biopsies were targeted after contrast injection immediately before tissue sampling. Multiple passes with the pediatric biopsy forceps through the catheter were possible, when needed. Biopsies were diagnostic in all patients where sampling was possible (4/4 patients). Immunohistochemistry was deemed necessary and successfully performed from the biopsies in 2/2 patients.

\section{Discussion}

We present technical aspects of the first clinical use of a novel triple lumen biopsy catheter for tissue diagnosis in small ducts (here: bile ducts with transpapillary and percutaneous access) that allows wire guidance and contrast application through the same catheter. We have previously used freehand biopsy or $11 \mathrm{Fr}$ pushing catheter sheaths (similar to a recent report [8]) to allow for easier passage of the biopsy forceps into the bile duct, if cholangioscopy was not immediately mandated. These accessories have a similar outer diameter to the catheter described here. With the novel design, we hope to address multiple issues: (1) freehand biopsy forceps introduction or positioning can be difficult and risky due to lack of guidance across the papilla and of navigation options inside the bile duct, especially 

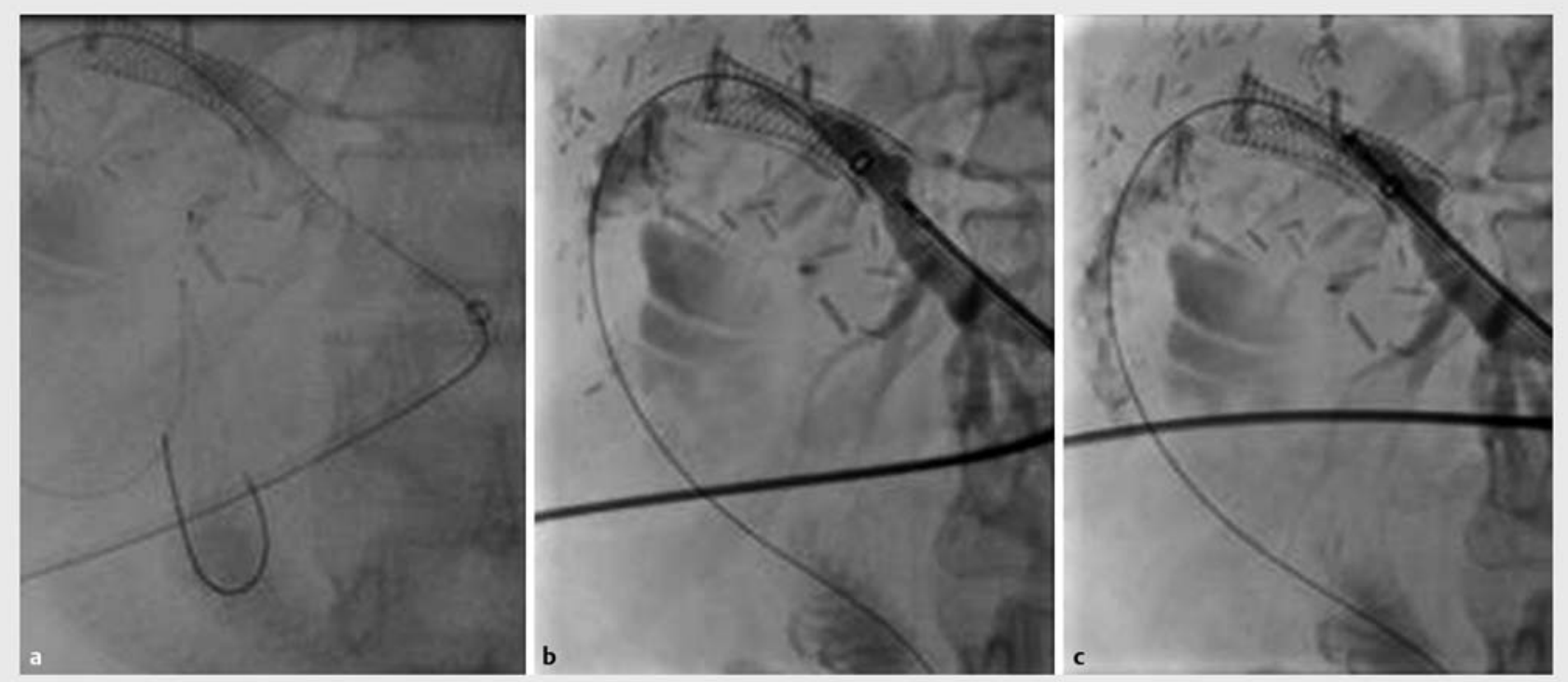

- Fig. 3 Percutaneous fluoroscopy-guided biopsies (left liver lobe access after hemihepatectomy for cholangiocellular carcinoma [CCC]). a After a guidewire is placed across the bilio-digestive anastomosis (bridged by a metal stent), the catheter (ring marker) is advanced percutaneously. $\mathbf{b}$ With the biopsy forceps already advanced inside the catheter, contrast injection delineates the in-stent stenosis. $\mathbf{c}$ A set of biopsies is obtained from the ingrowing tissue of the in-stent stenosis.

if multiple passes are required. Here, the triple lumen catheter serves as a sheath across the papilla and is positioned inside the bile duct following the guidewire. (2) Contrast usually drains rapidly from the bile duct once sphincterotomy is performed and an accessory introduced. With the catheter, reinjection of contrast can be performed easily without changing instruments. (3) Brushing often yields inadequate specimens, and biopsies from cholangioscopy are fairly small. Here, we used a $1.8-\mathrm{mm}$ standard pediatric biopsy forceps. (4) Though cholangioscopy with biopsy will probably remain the diagnostic gold standard, costs of the novel catheter will be far less than costs associated with cholangioscopic sampling.

There are clear limitations inherent to our current data: We failed to introduce the catheter in one patient and could not navigate toward a stenosis in the proximal left hepatic duct due to the fairly stiff prototype material. This can be easily dealt with in a follow-up design. Secondly, we did not aim to evaluate the diagnostic performance in terms of biopsy accuracy compared with final diagnosis in this first setting, but only technical aspects. This will be addressed in a follow-up trial. Thirdly, although we did not see any adverse events, the number is far too low to base any reasonable calculation on the current data. However, we have good reason to believe that a catheter design with targeting option is at least no more prone to adverse events than freehand biopsies from the bile ducts.

In the current design, the working channel can host any accessory suitable to be introduced through it, such as biopsy forceps, but also stone baskets, balloons, or visualization (probebased confocal laser endomicroscopy [pCLE]) and therapeutic (electrohydraulic lithotripsy (EHL), photodynamic therapy [PDT]) probes. In addition, catheter use is not limited to bile ducts. By analogy, wire-guided use in the pancreatic duct, ureter or other small hollow structures is conceivable.

In summary, we present encouraging technical results using a novel prototype triple lumen catheter for large volume biopsy under contrast and wire guidance. The catheter is simple to use and potentially relatively inexpensive. Clinical usefulness in comparison to standard of care will have to be established subsequently.

\section{Competing interests}

A patent for the catheter has been filed by the author (MG), the Department of Gastroenterology (Innere Medizin I), and the University of Tübingen (PCT/EP2016/070530).

\section{References}

[1] Korc P, Sherman S. ERCP tissue sampling. Gastrointest Endosc 2016; 84: $557-571$

[2] Ponchon T, Gagnon P, Berger F et al. Value of endobiliary brush cytology and biopsies for the diagnosis of malignant bile duct stenosis: results of a prospective study. Gastrointest Endosc 1995; 42: 565 572

[3] Schoefl R, Haefner M, Wrba F et al. Forceps biopsy and brush cytology during endoscopic retrograde cholangiopancreatography for the diagnosis of biliary stenoses. Scand J Gastroenterol 1997; 32: 363-368

[4] Pugliese V, Conio M, Nicolo G et al. Endoscopic retrograde forceps biopsy and brush cytology of biliary strictures: a prospective study. Gastrointest Endosc 1995; 42: 520 - 526 
[5] Nishikawa T, Tsuyuguchi T, Sakai Y et al. Comparison of the diagnostic accuracy of peroral video-cholangioscopic visual findings and cholangioscopy-guided forceps biopsy findings for indeterminate biliary lesions: a prospective study. Gastrointest Endosc 2013; 77: 219-226

[6] Ogura T, Imanishi M, Kurisu Y et al. Prospective evaluation of digital single-operator cholangioscope for diagnostic and therapeutic procedures (with videos). Dig Endosc 2017; 29: 782 - 789
[7] Rey JW, Hansen T, Dumcke S et al. Efficacy of SpyGlass(TM)-directed biopsy compared to brush cytology in obtaining adequate tissue for diagnosis in patients with biliary strictures. World J Gastrointest Endosc 2014; 6: 137-143

[8] Morita S, Kanefuji T, Hoshi T et al. A novel technique for biliary biopsy using the sheath of a plastic stent and a non-return valve. Endoscopy 2017; 49: E9-E10 